
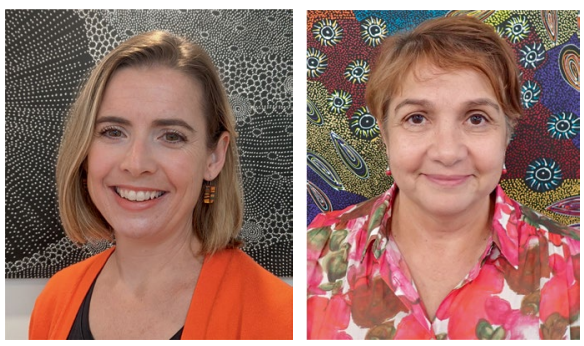

Credit: Left and right images courtesy of William Radford and Zyana Gall, respectively

boriginal and Torres Strait Islander people, the Indigenous peoples of Australia, account for $3.3 \%$ of the total population. Although relatively young in age structure compared to nonIndigenous Australians, the Indigenous population is aging rapidly, with its oldest members projected to increase threefold in number over the next 30 years. Indigenous Australians are diverse, live in a wide variety of environments and circumstances, and carry forward some of the longest continuously surviving and dynamic cultures in the world. Older Indigenous people contribute cultural custodianship, caregiving and leadership to their communities. Population aging is welcome, but ensuring the health and quality of life of older Indigenous peoples is vital.

Indigenous Australians face substantial health inequities that are deeply rooted in the legacy of colonization and ongoing marginalization. This systemic disadvantage is evident in lower average life expectancy of almost a decade compared to nonIndigenous Australians, and significant disparities in rates of chronic physical and mental health conditions, disability and disease outcomes. Moreover, dementia rates are among the highest observed globally at up to $24 \%$ of Indigenous Australians aged 60 years and older. The health impacts of intergenerational trauma and racial discrimination are well-documented and remain at the forefront of everyday experience for many Indigenous people. Within this population, manifold relative disadvantage experienced by Stolen Generation survivors (who endured forced childhood removal and institutionalization and are all eligible for aged care by 2023), recently highlighted by the Healing Foundation (https://bit.ly/3vff1k0), is a clear example of the direct impact that government policies have had on population health and aging outcomes.

Many Indigenous-led public health initiatives are proving effective, seeing

\title{
Sovereignty at the heart of aging well for Australia's First Nations
}

\author{
Indigenous Australians, one of the oldest living civilizations in the world, \\ are growing older despite centuries of health and social inequity. Further \\ improvements in longevity and aging will require a life-course approach and \\ community-led initiatives.
}

rates of smoking decline ${ }^{1}$, improved access to cancer screenings ${ }^{2}$ and childhood immunization rates soaring to $97.26 \%$ of 5-year-olds ${ }^{3}$. Throughout the COVID-19 pandemic, Indigenous-led initiatives to protect communities, particularly Elders and older people, are notable for their success $^{4}$ and have contributed to Indigenous Australians experiencing very low infection rates ( $0.5 \%$ of cases in Australia) and no deaths related to COVID-19 (ref. ${ }^{5}$ ).

Comprehensive, sustained efforts are needed to further improve health, longevity and quality of life with and for this aging population. Developing shared principles and priorities to guide efforts to improve aging outcomes is critical. Australia's National Health and Medical Research Council now acknowledge healthy aging and dementia as priority areas for Indigenous health research, and have targeted investment in this area. The recent Royal Commission into Aged Care Quality and Safety, which was instigated following mounting public concern regarding Australia's aged care system and institutions, including abuse and neglect of older people, sought extensive evidence in relation to Indigenous aged care issues. The Commission's final report ${ }^{6}$ made specific recommendations regarding Indigenous aged care; however, there is currently no detailed plan for implementation. Older Indigenous people have witnessed dramatic social change in their lifetime, including the origination and growth of the community-controlled health sector and a rise in Indigenous representation across many spheres of public life. However, it is distressing to also see repeated national policy failures - notably, the Closing the Gap policy ${ }^{7}$ - for addressing Indigenous health and social disparities, and rising rates of child removal, incarceration and deaths in custody in the years since landmark inquests into these issues from the 1990s. Social and political reform is overdue in Australia, as has been offered through the 2017 Uluru Statement from the Heart ${ }^{8}$.

Healthy aging frameworks, policy and programs must be grounded in Indigenous understandings of health and wellbeing. Indigenous Australians commonly embrace holistic and collectivist conceptions of health that include the wellbeing of the individual and community. However, for older Indigenous people and their families, access to culturally responsive services is often limited. Appropriate local models of care and care choices are required, but the transition to market-based, consumerdirected aged care funding in Australia has widely served to exacerbate issues with inequitable access. Urgent action is needed to build a culturally competent aged care workforce and to design culturally appropriate systems and services. This requires substantial government investment, particularly for Aboriginal communitycontrolled organizations and peoples to develop skills and services to support aging communities. Interventions need to be developed, implemented and evaluated in partnership with Indigenous communities, with outcome measures reflecting aspects of life that are relevant to and valued by them. Indigenous leadership, community empowerment and authentic partnerships are crucial to enhancing aged care access, quality and cultural safety.

Finally, Australia (and, indeed, the global community) needs a life-course, population health approach to aging well, with focus on reducing social inequity and systemic racism, to reduce the burden of age-related health conditions, including cancer, frailty and dementia. The foundations for aging well are established in the earliest phases of life and are supported by loving families; strong, connected and safe communities; culture and enriching experiences; access to high-quality education and healthcare; and food, housing and income security. The survival, resilience and now aging of Indigenous Australians is remarkable in the 
context of Australia's devastating settlercolonial history, but the continued fight for mere parity in health and longevity should not and cannot be borne by Indigenous peoples alone. Closing the gap and achieving healthy aging for Indigenous Australians requires Indigenous-led responses that emphasize self-determination, supported by all Australians.

\section{Kylie Radford (D) 1,2,3凶 and Gail Garvey (D) 4,5,6}

${ }^{1}$ Neuroscience Research Australia (NeuRA), Sydney, New South Wales, Australia. ${ }^{2}$ School of Psychology, University of New South Wales, Sydney, New South Wales, Australia. ${ }^{3}$ Ageing Futures Institute, University of New South Wales, Sydney, New South Wales, Australia. ${ }^{4}$ Menzies School of Health Research, Charles Darwin University, Brisbane, Queensland, Australia. ${ }^{5}$ School of Public Health, University of Queensland, Brisbane, Queensland, Australia. ${ }^{6}$ St Vincent's Health Australia, Brisbane, Queensland,
Australia.

凶e-mail:k.radford@neura.edu.au

Published online: 15 July 2021

https://doi.org/10.1038/s43587-021-00084-w

References

1. Bain, C. Smoking rates for Indigenous Australians are dropping faster than the general population. SBS News https://www.sbs. com.au/news/smoking-rates-for-indigenous-australians-aredropping-faster-than-the-general-population (2020).

2. National Indigenous Bowel Screening Pilot. Australian Government Department of Health https://www.health.gov.au/initiatives-andprograms/national-indigenous-bowel-screening-pilot (2021).

3. Immunisation coverage rates for Aboriginal and Torres Strait Islander children. Australian Government Department of Health https://www.health.gov.au/health-topics/immunisation/ childhood-immunisation-coverage/immunisation-coveragerates-for-aboriginal-and-torres-strait-islander-children (2021).

4. Keene, M. COVID-19 and Indigenous Australians: a chronology. Research Papers 2020-21 (Department of Parliamentary Services, Parliament of Australia, 2020); https://www.aph.gov.au/About Parliament/Parliamentary_Departments/Parliamentary_Library/ pubs/rp/rp2021/Chronologies/COVID19-IndigenousAustralians

5. Power, J. When it came to COVID-19, Indigenous Australians sent it packing. The Sydney Morning Herald https://www.smh. com.au/national/when-it-came-to-covid-19-indigenousaustralians-sent-it-packing-20201112-p56e5u.html (2020).

6. Final Report: Care, Dignity and Respect (Royal Commission into Aged Care Quality and Safety, 2021); https://agedcare. royalcommission.gov.au/publications/final-report

7. A Ten-year Review: the Closing the Gap Strategy and Recommendations for Reset (Australian Human Rights Commission, 2018); https://bit.ly/3gonYCp

8. The Uluru Statement From the Heart. The Uluru Statement https://ulurustatement.org/the-statement (2021).

\section{Acknowledgements}

We acknowledge the Aboriginal Health and Ageing Program team at Neuroscience Research Australia (NeuRA) for their comments on early drafts of this article, and for their contributions to understanding and promoting aging well in partnership with Aboriginal communities.

Author contributions

The authors declare that they contributed equally to this manuscript.

Competing interests

The authors declare no competing interests. 